\title{
ONTOLOGY DEVELOPMENT IN HOME AUTOMATION USED IN AUTOMATIC VE FORMATION
}

\author{
Orlando Ribas Fernandes, Ana Paula Rocha, Eugénio Oliveira \\ LIACC - NIAD\&R, Faculty of Engineering, University of Porto \\ R. Dr. Roberto Frias, 4200-465 Porto, Portugal \\ orlando.ribas@gmail.com, arocha@fe.up.pt, eco@fe.up.pt
}

\begin{abstract}
Ontologies are crucial in B2B electronic commerce in general and in the Virtual Enterprise scenario in particular, enabling the mutual understanding between multiple business participants. This paper considers the development of a specific ontology in the home automation business area, illustrated in the Virtual Enterprise formation process. The ontology developed for the home automation (domotics) domain enumerates different products, its attributes, mutual relations and implicit business rules. A case study is presented based on a real scenario where business participants are represented by multiple agents in the electronic market.
\end{abstract}

\section{INTRODUCTION}

The main goal of the research project behind this paper was the development of a specific ontology in the home automation business area, illustrated in the Virtual Enterprise formation scenario.

The Virtual Enterprise (VE) is a flexible organizational structure, composed by autonomous and individual enterprises committed between them in temporary associations in order to answer new market requirements (Fischer et al., 1996) (Norman et al., 2004). Since these individual enterprises are heterogeneous and probably unknown to each other, the regularization of its joint activity is a crucial issue. This task can be effectively done by the so called Electronic Institution (EI), by imposing norms and rules on the cooperative behaviour of their participants.

In our approach, the EI is modelled through an open Multi-Agent System (MAS), where agents represent both enterprises and customers of the electronic market (Cardoso et al. 2005). Like the institutions that regulate the way citizens interact in human societies, the EI concept represents the virtual counterpart of real-world institutions (Arcos et al., 2005) (Cardoso et al. 2005). Besides that normative framework, the EI also provides a set of institutional services that helps in the effective coordination between agents which represent different entities of the real-world, and interact for the establishment of business relationships. These institutional services include: i) appropriate negotiation algorithms enabling the selection of the most suitable VE partners according to current needs (Rocha et al. 2005); ii) the establishment and monitoring of electronic contracts (Cardoso et al. 2005), and iii) ontology services that ensure the interoperability between heterogeneous agents with their own languages and ontologies (Malucelli and Oliveira, 2005). Although the EI also includes ontology services, these will not be used in the present work, since it 
is not the goal's work. In this paper, we will therefore assume that agents have similar ontologies.

We intend to use our Electronic Institution framework with a specific ontology developed in the home automation area, to illustrate the VE formation stage. The developed ontology includes the enumeration of different products of the home automation domain, its attributes, relations and the implicit business rules.

The rest of the paper will unfold as follows. In section 2 we discuss the ontology concept empathizing on the ontology development process. In section 3, we present the Virtual Enterprise concept. Next, section 4 describes an ontology implementation in the home automation domain, and presents a case study where this ontology is used in our framework to illustrate the Virtual Enterprise formation process. Finally, section 5 presents our conclusions.

\section{ONTOLOGIES}

Ontology is a popular topic in the Artificial Intelligence domain with relevance in areas like: Knowledge Engineering, Natural Language and Multi-Agent Systems. But ontologies aren't limit to the Artificial Intelligence domain, being also applied, for example, in the community World Wide Web (WWW).

In the area of the computer science many and arguable definitions exist for the word "ontology". The more widely accepted in the context of Artificial Intelligence is the one of (Gruber, 1993) that defines ontology as an explicit specification of a conceptualisation. "Conceptualisation" is basically the idea of the world that a person or a group of people may have; "explicit" means that the type of concepts and restrictions about their use are explicitly defined.

Another very accepted definition in the scientific community is the one of (Uschold and Jasper, 1999) which states that, although ontologies may have several forms, always includes a specific vocabulary and some specifications as far as their meaning is concerned. Ontologies include definitions and relations of how the concepts are interrelated, which imposes a collective domain structure and restricts the possible interpretations of the existing terms.

Several studies and methodologies exist regarding the ontology development task. According to these studies, the ontology development can be decomposed in four steps (Denny, 2002):

- Domain knowledge acquisition: Sources of knowledge and expertise are collected in a structured way in order to formally describe the domain knowledge.

- Ontology organization: Identification of the main concepts related to the domain, its properties. Relations between concepts are also identified.

- Ontology instantiation: Individual instances are added following the concepts and relations already named.

- Verification: Ontology syntax and semantic is verified (sometimes in an automatic way) in order to detect and correct inconsistencies.

The ontology developed and reported in this work follows the issues just mentioned. 


\section{VIRTUAL ENTERPRISE}

Ontologies are crucial in B2B electronic commerce in general, and in Virtual Enterprise in particular, enabling the mutual understanding between multiple business participants. A Virtual Enterprise (VE), according to Fischer (Fischer et al., 1996), is defined as a voluntary cooperation of some autonomous and heterogeneous enterprises that are, possibly, geographically distributed. All enterprises that are members of the cooperation provide its resources, abilities, capacities and knowledge, for the attainment of a service or product with base in a common agreement. The VE is also an agile structure, and so has the ability to dynamically reconfiguration in reaction to environment changes.

This new concept of enterprise entity enables the reduction of individual enterprises' processes for main abilities that try to get with the maximum quality and profit. Thus, individual enterprises can become a niche of ability in a specific area of its domain. The formation of corporations allows the share of such abilities, costs, resources (of capital, intellect and technological), and still the reduction of the complexity of the problem.

The VE life cycle comprises four phases (Fischer et al. 1996):

- Identification of needs: Appropriate description of the product or service to be delivered by the VE, which guides the conceptual design of the VE;

- Formation (partners selection): Automatic selection of the individual enterprises (partners), which, based in its specific knowledge, skills, resources, costs, and availability, will integrate the VE;

- Operation: Control and monitoring partners' activities, including resolution of potential conflicts, and possible VE reconfiguration due to partial failures;

- Dissolution: Breaking up the VE, distributing the obtained profits, and storing relevant information for future use.

Although not being the focus of this paper, it should be said that our framework includes a specific negotiation protocol to select the participants that, based on its capabilities and availability, will be able to make the optimal deal according to its own goals (the second phase of the VE life cycle). This is an iterative multi-attribute negotiation between potential VE partners and the VE organizer that includes a reinforcement learning capability in the formulation of participants' counter-proposals (Rocha et al, 2005).

In this paper, we will illustrate, based in a real business scenario, how the VE formation can be accomplished using our own framework.

\section{CASE STUDY IN HOME AUTOMATION}

In our scenario, Enterprises and Customer Agents meet in a market place where they cooperate to form a Virtual Enterprise with the objective of supply/get goods according to their own goals and preferences. These goods under negotiation are described by each agent using their private ontologies. As mentioned before, in this work the agents use the same ontology. The current version of our EI platform does not include the ontology services, although this module is currently being integrated in it.

The ontology developed in the current work is related with the home automation area. For its development, we selected the Protégé tool (Protégé 3.2, 2007) as the ontology editor. This choice is justified by the high usability of Protégé that offers an excellent development environment, with multiple third parties plug-ins that allows the use of several services for varied ends. 
The language chosen for the ontology representation was Ontology Web Language (OWL). The OWL is a marking language for definition, publication and allotment of ontologies in the Internet, and is recommended by the W3C ${ }^{1}$ since 2004. Being OWL based on XML, gets all the advantages of this language, stands out the fact that the information can also be changed between different types of systems (operative systems and languages of applications).

\subsection{Home Automation Ontology}

The first step on the ontology development process is the "Domain Knowledge Acquisition". The data supplied by the domain experts is analysed and discussed with them which allows knowing in depth the domain of interest. In this work, the data was supplied by a home automation company, "Central Casa".

The second step on the ontology development process is the "Ontology Organization". The data collected in previous step is organized into concepts (classes) that specify the products commercialized by the home automation company.

Figure 1 illustrates the structuralized organization of the created concepts (classes), using the Protégé tool. It is understood that the Products class would be the superclass of the existing products in the data received from the company. These products concern cameras, remote controls, micromodules, X10 modules and security equipment. Packs class will contain a set of other products.

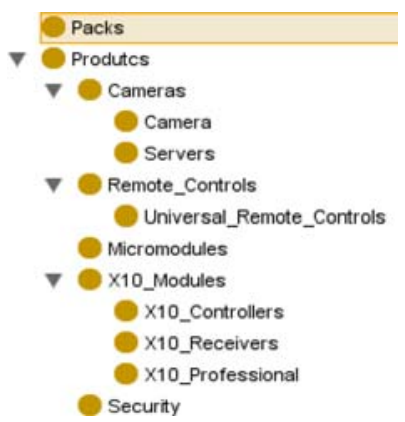

Figure 1. Ontology classes

In an ontology, properties or attributes can be of two types: Object properties or Datatype properties. Object properties connect one instance to another, and Datatype properties assign a value to an instance. In this ontology, we consider both Object and Datatype properties that are the following: Applications, Characteristics, Description, Informations, Price, Quantity, Ref and belongPack..

The properties Applications, Characteristics, Description, Informations and Ref are Datatype properties. The necessity to include these properties was found during the data analysis process. Products commercialized by the company had several textual information related to different issues, which are now enumerated in these properties.

The properties Price and Quantity are also Datatype properties. This information did not exist in the data received originally, but it was added as a necessity to the electronic negotiation that will go to elapse in the process of the VE formation.

The property belongPack is an Object property. It is used to indicate that an instance belong to a specific pack (Packs is a concept of this ontology).

\footnotetext{
${ }^{1}$ The recommendations of $\mathrm{W} 3 \mathrm{C}$ are understood by the industry and the scientific community as standards Web
} 
The next step in the ontology development process is the "Ontology Instantiation", where concepts considered in the ontology are now instantiated. As an example of it, Figure 2 illustrates the instantiation of the concept "Packs".

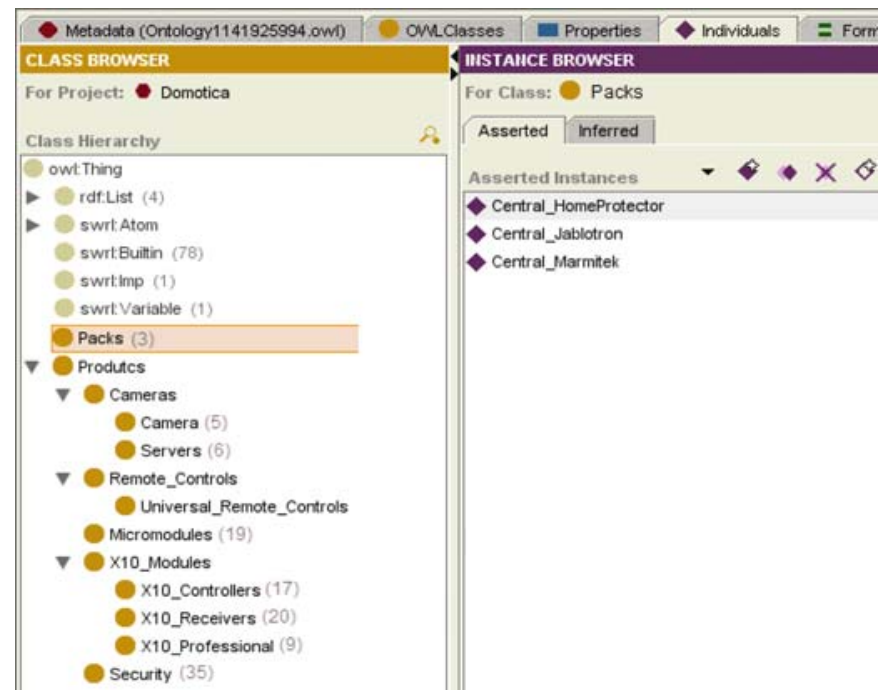

Figure 2. "Packs" instantiation

In this step, properties and relations are also filled in. Figure 3 illustrates the property belongPack in a particular case where two instances (Motion_detector and Smoke_detector) are considered elements of the pack Central_Homeprotector.

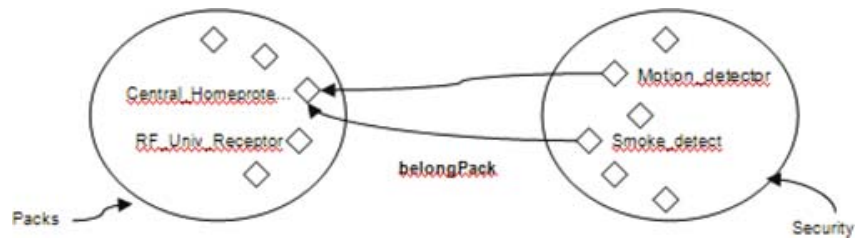

Figure 3. Relations between instances

The final step in the ontology development process, "Verification", was done in conjunction with the domain experts through a manual analysis of the developed ontology. Finally, the ontology was saved in OWL format, in order to be used by our EI framework.

\subsection{Case Study Discussion}

The following stage of the project consisted on the addition of a new module to the Electronic Institution platform, which allows the creation, edition and storage of proper ontologies on the intervening agents, so that they can be used on future interactions.

In the current platform, each agent in the EI has its own window. In this window it is possible to configure the agent abilities (if it is an enterprise agent that sells goods) or needs (if is a client agent that wants to buy goods), as well as to visualize its activity in the market. 
The problem of VE formation is, essentially, a problem of competitive negotiation, where the different enterprise agents compete among themselves for the selection (by the client agent) as members of the VE. The negotiating strategy of each enterprise agent is the adoption of the action plan that leads the agent to his objective, which usually translates into maximizing its utility function. Enterprises and client agents will engage in a round of proposal and counterproposals. In each round, enterprises agents try to improve their proposals in order to beat concurrent enterprises and be selected by the client agent. The trading ends when the client agent receives an adequate proposal (a proposal that has a utility value higher than a pre-defined threshold), or when a time limit is reached.

The negotiation process that leads to the VE formation is done through the negotiation of the values of some properties of the instances in discussion. The values of these properties (the ones that are negotiable) can have two ranges: the domain values and the preferable values. The domain values are the ones that the agent accepts during the negotiation. The preferable values are the one the agent will prefer among others in discussion (the preference values are a subset of the domain values)

In the scenario presented, the client wants to equip its house with a safety system for detecting intruders and detecting fire. The correct solution to this particular client is the product Central_Marmitek pack as defined in the ontology. This product is composed by other five products: Remote_Control-MaxiControl, Alarm-HomeGuard, Smoke_detectorMaxiControl,Pocket_Control-MaxiControl and Sirene_PowerHorn-MaxiControl.

In this scenario the client agent (named Central_Casa) is interested in five products. The process of selecting partners for the VE includes, therefore, five parallel negotiations, one for each product. In each of these negotiations are involved different enterprise agents (Ent_Forn_1 to Ent_Forn_10) with responsibility for the provision of the respective component. Each enterprise agent assigns different domain values to the negotiable properties

We consider as negotiable properties the Quantity and Price properties. The domain and preferable values that the Central_Casa agent assigns to the negotiable properties are enumerated in Table1. In this case, is specified a large range for the attribute Quantity, in order to better analyze the behavior of each agent in the negotiation process.

Table 1. Preferred Values - Cental_Casa Agent

\begin{tabular}{|c|c|c|}
\hline Components & Atributes & Preferred \\
\hline \multirow{2}{*}{$\begin{array}{c}\text { Remote_Control- } \\
\text { MaxiControl }\end{array}$} & Quantity & $1-3000$ \\
\hline & Price & $1-35$ \\
\hline \multirow{2}{*}{$\begin{array}{l}\text { Alarm- } \\
\text { HomeGuard }\end{array}$} & Quantity & $1-3000$ \\
\hline & Price & $1-70$ \\
\hline \multirow{2}{*}{$\begin{array}{c}\text { Smoke_detector- } \\
\text { MaxiControl }\end{array}$} & Quantity & $1-3000$ \\
\hline & Price & $1-60$ \\
\hline \multirow{2}{*}{$\begin{array}{c}\text { Pocket_Control- } \\
\text { MaxiControl }\end{array}$} & Quantity & $1-3000$ \\
\hline & Price & $1-50$ \\
\hline \multirow{2}{*}{$\begin{array}{l}\text { PowerHorn } \\
\text { MaxiControl }\end{array}$} & Quantity & $1-3000$ \\
\hline & Price & $1-75$ \\
\hline
\end{tabular}

After the negotiation, limited in this scenario to 5 rounds, the client agent Central_Casa selected the VE partners Ent_Form_2, Ent_Form_1, Ent_Form_1, Ent_Form_9 and Ent_Form_3 to supply the products Comando Remoto, Alarme HomeGuard, Sensor de Fumo - MaxiControl, Comando de Bolso - MaxiControl, Sirene PowerHorn - MaxiControl respectively. These partners were selected because they presented the best proposals concerning the negotiable properties Quantity and Price 
when the negotiation ended. Figure 4 shows the utility value obtained by the client agent with the proposals of each one of the selected partners.

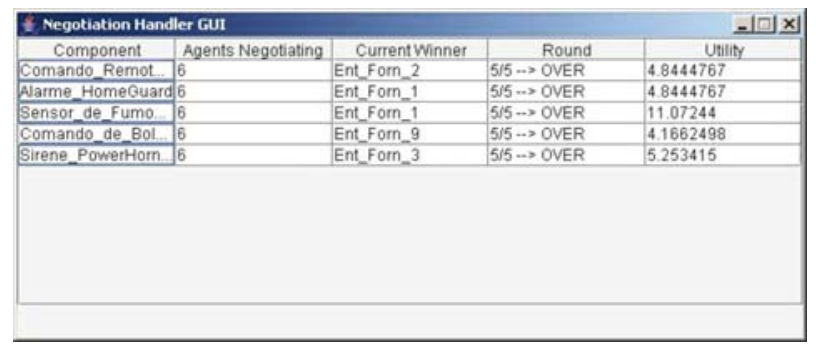

Figure 4. Agents that are in VE

\section{CONCLUSIONS}

Due to the necessity to get competitive advantage in the electronic market, it becomes crucial to make better and different than competitors, and mainly to have the capacity to create the single value for customers. It was considered, as a main goal of this work, the presentation of a realistic case in the home automation area, related to the Virtual Enterprise formation process. Thus, and with the support of the CentralCasa home automation company, ontology was developed in the business area of the home automation. We used the Protégé tool in the ontologies edition and modulation. The VE formation is one of the main phases of the VE life cycle, and an adaptive multi-attribute negotiation protocol (presented in (Rocha et al., 2005)) was used in the scenario presented here.

The already existing Electronic Institution platform was updated with the capacity to read and recognize ontologies developed using the Protégé tool. These ontologies are written in OWL.

This work can be improved in the future by including new functionalities in the current EI platform, namely the use of commitments and social intentions as well as trust and reputation mechanisms. These two topics are of great interest in B2B e-commerce in general, and VE scenario in particular, and are currently under development. Other future topics of research include:

- The development of more specific ontologies in the home automation area, and the inclusion of real Central Casa suppliers;

- The development of ontology services capable to support the formation and monitoring of the VE in an electronic market. Such services include generic processes for the creation of specific ontologies and services of translation for pre-existing ontologies.

\subsection{Acknowledgments}

We thank CentralCasa home automation company for providing the data used in our scenario. 


\section{REFERENCES}

1. Cardoso HL, Malucelli A, Rocha AP, Oliveira E. "Institutional Services for Dynamic Virtual Organizations". In Collaborative Networks and Their Breeding Environments $-6^{\text {th }}$ IFIP Working Conference on Virtual Enterprises, L.M. Camarinha-Matos, H. Afsarmanesh, A. Ortiz (eds.): Springer, Valencia, Spain, 2005: 521-528.

2. Denny, Michael. "Ontology Building: A Survey of Editing Tools”, Technical report, O’Reilly XML.com, 2002.

3. Fischer K, Muller JP, Heimig I, Scheer A. "Intelligent Agents in Virtual Enterprises". In $1^{\text {st }}$ International Conference on the Pratical Application of Intelligent Agents and Multi-Agent Technology, London, UK, 1996.

4. Gruber, Thomas R.. "Toward Principles for Design of Ontologies Used for Knowledge Sharing”. In N. Guarino, R. Poli (eds.), Formal Ontology in Conceptual Analysis and Knowledge Representation, Kluwer Academic Publishers, 1993.

5. Malucelli A., Oliveira E. "Using Similarity Measures for an Efficient Business Information-Exchange". In IEEE/WIC/ACM International Conference on Intelligent Agent Technology (IAT 2005), Compiègne, France, 2005.

6. Malucelli, A., Oliveira, E. "Ontology-Services Agent to Help in the Structural and Semantic Heterogeneity". In Virtual Enterprises and Collaborative Networks, L. M. Camarinha-Matos (ed.), Kluwer Academic Publishers, 2006: 175-182.

7. Norman TJ, Preece A, Chalmers S, Jennings NR, Luck M, Dang D, Nguyen TD, Deora V, Shao J, Gray A. Fiddian N. "Agent-based formation of virtual organisations". In Int. J. Knowledge Based Systems 17, 2004: 103-111.

8. Uschold M, Jasper RA. "Framework for Understanding and Classifying Ontology Applications". In VR Benjamins (ed.), IJCAI'99 Workshop on Ontology and Problem Solving Methods: Lessons Learned and Future Trends (KRR5), Stockholm, Sweden, 1999.

9. Arcos JL Marc Esteva, Noriega P, Rodriguez-Aguilar JA, Sierra C. "Engineering open environments with electronic institutions". In Engineering Applications of Artificial Intelligence, 2005: 18:191-204.

10. Protégé 3.2. The Protégé Ontology Editor and Knowledge Acquisition System, Stanford Center for Biomedical Informatics Research, Stanford University School of Medicine, 2007.

11. Rocha AP, Cardoso HL, Oliveira E. "Contributions to an Electronic Institution supporting Virtual Enterprises' life cycle”. In Virtual Enterprise Integration: Technological and Organizational Perspectives, G. D. Putnik \& M. M. Cunha (eds.), Idea Group Inc., 2005: 19:229-246. 